\title{
Effect of statins on coronary blood flow after percutaneous coronary intervention in patients with stable coronary artery disease
}

\author{
L. Cerit ${ }^{1}$ H. Duygu ${ }^{1} \cdot$ K. Gulsen ${ }^{1} \cdot$ A. Gunsel ${ }^{1}$
}

Published online: 25 August 2016

(C) The Author(s) 2016. This article is available at SpringerLink with Open Access.

\begin{abstract}
Aims Statins have favourable effects on the vascular system. However, few data are available regarding the effect of these drugs on patients undergoing percutaneous coronary intervention (PCI). We sought to determine the impact of prior statin use on coronary blood flow after PCI in patients with stable coronary artery disease (CAD) by using the corrected thrombolysis in myocardial infarction (TIMI) frame count (CTFC).

Methods A total of 80 consecutive eligible patients (mean age: $60 \pm 7$ years, $65 \%$ male) with the diagnosis of stable CAD who were hospitalised for elective PCI were retrospectively enrolled in our study. The study population was divided into two groups according to statin use at least 6 months before PCI. Group 1 comprised of 51 patients (67\% male; mean age: $58 \pm 4$ years) taking statins and group 2 comprised of 29 patients (62\% male; mean age: $60 \pm 3$ years) not taking statins. PCI was applied to de novo type A lesions. CTFC was calculated for the treated vessels at baseline and after PCI.

Results The two groups had similar characteristics in terms of age, sex, concomitant medications, lesion characteristics, pre-procedural CTFC, lipid parameters, and risk factors for CAD. Post-PCI CTFC (16 \pm 3 vs. $22 \pm 5, p=0.01)$ and hs$\mathrm{CRP}(2.1 \pm 0.7 \mathrm{mg} / \mathrm{l}$ vs. $6.1 \pm 2 \mathrm{mg} / \mathrm{l}, p=0.01)$ in patients receiving statins before PCI were significantly lower than in patients without statin therapy. Multiple logistic regression analysis showed that statin pre-treatment (OR 2.5, $95 \% \mathrm{CI}$ 1.2 to $3.8, p<0.001$ ) and hs-CRP level (OR 1.8, $95 \% \mathrm{CI}$
\end{abstract}

H. Duygu

hamzakard@yahoo.com

1 Department of Cardiology, Near East University Hospital, Nicosia, Cyprus
1.2 to $2.4, p=0.001)$ were independent predictors of postPCI CTFC.

Conclusions In patients with stable CAD undergoing PCI, receipt of long-term statin therapy was associated with improvement in epicardial perfusion after PCI.

Keywords Statin - Coronary blood flow - Stable coronary artery disease

\section{Introduction}

Cholesterol reduction with HMG-CoA (3-hydroxy-3methylglutaryl coenzyme A) reductase inhibitors or statins has been shown to improve mortality and cardiovascular morbidity in patients with established coronary artery disease (CAD) [1, 2]. Recent studies suggest that the incidence of early death and recurrent ischaemic events is also reduced by statin therapy in acute coronary syndrome (ACS) patients [3, 4]. However, few data are available regarding the effect of these drugs on patients undergoing percutaneous coronary intervention (PCI). Previous evidence suggests that statins have various favourable effects on the vascular system that are not directly related to their impact on lipid metabolism. Beyond lowering lipids, statins have favourable effects on platelet adhesion, thrombosis, endothelial function, plaque stability, and inflammation [5-8]. As with ACS, the vascular injury from coronary angioplasty and stent placement induces platelet activation, thrombosis, and inflammation within the vessel wall and the distal microvasculature. Therefore, in addition to a long-term benefit associated with lipid lowering, statin therapy might play a beneficial role early after PCI.

The thrombolysis in myocardial infarction (TIMI) frame count (TFC) is a simple clinical tool for assessing quantita- 
tive indexes of coronary blood flow. The TFC is objective, quantitative, reproducible, and sensitive to changes in coronary flow [9]. It has been suggested that a higher TFC may reflect disordered resistance vessel function or microvascular dysfunction [9]. To our knowledge, there are no reports regarding the effect of prior statin use on coronary blood flow in patients with stable CAD undergoing PCI. We sought to determine the impact of prior statin use on coronary blood flow after PCI in patients with stable CAD by using TFC.

\section{Methods}

\section{Study population}

A total of 80 consecutive eligible patients (mean age: $60 \pm$ 7 years, $65 \%$ male) with the diagnosis of stable CAD and who were admitted for elective PCI were retrospectively included in our study. The study population was divided into two groups according to statin use at least for 6 months before PCI. Group 1 comprised 51 patients (67\% male; mean age $58 \pm 4$ years) taking statins for at least 6 months before PCI and group 2 comprised 29 patients (62\% male; mean age: $60 \pm 3$ years) not taking statins. Statin treatment was started $10 \pm 2$ months prior to the PCI. The type and dosage of the statins used were as follows: (1) atorvastatin $79 \%$ of cases (mean dose $30 \pm 10 \mathrm{mg} /$ day); (2) pravastatin, $11 \%$ of cases (mean dose $20 \pm 10 \mathrm{mg} /$ day); and (3) simvastatin, $10 \%$ of cases (mean dose $25 \pm 10 \mathrm{mg} /$ day).

PCI was performed based on the ongoing symptoms (refractory angina) despite optimal medical therapy. Chronic stable angina was defined as effort angina with objective ischaemic evidence on thallium scintigraphy or exercise testing over the past 6 months. Hypertension was defined as a blood pressure of $140 / 90 \mathrm{~mm} \mathrm{Hg}$ or more or treatment with antihypertensive medication, current cigarette smoking as active smoking, and family history of CAD if patients had a first-degree male relative with evidence of $\mathrm{CAD}<55$ years of age or a female relative $<65$ years of age. The body mass index was also measured. Exclusion criteria were: acute coronary syndromes; severe hepatic or renal dysfunction; diabetes mellitus; malignant or inflammatory diseases; local or systemic infection; anti-inflammatory drug use; and complex coronary lesions (total occlusions, highly calcified lesion, left main coronary artery lesion, restenosis, and vein graft lesion).

\section{Adjunctive pharmacotherapy}

All patients received $100 \mathrm{mg}$ per day of acetylsalicylic acid before intervention and unfractionated heparin during PCI on a routine basis. Clopidogrel was given in accordance with the guidelines. The glycoprotein IIb/IIIa inhibitors were administered at the discretion of the operator. Unfractionated heparin bolus at $100 \mathrm{IU} / \mathrm{kg}(50-70 \mathrm{IU} / \mathrm{kg}$, if glycoprotein IIb/IIIa receptor inhibitor is administered) was given to all patients and the periprocedural activated clotting time (ACT) was measured. Unfractionated heparin dosage was adjusted under ACT guidance (ACT in the range of 250-300 or 200-250 s, if glycoprotein IIb/IIIa receptor inhibitors are given).

\section{Angiographic analysis}

Selective coronary angiographic examinations were performed by the standard Judkins technique. The coronary diameters and stenosis percentages were measured by computerised quantitative angiography in a biplane mode. Angiographic assessment was always performed by two independent angiographers blinded to the patients' clinical data.

Only bare-metal stents with or without balloon predilatation were used in this study. PCI was considered successful if the final percent diameter stenosis was $<50 \%$, with TIMI grade 3 flow in the absence of recurrent ischaemia, myocardial infarction, need for bailout stenting or urgent coronary bypass surgery during hospitalisation, or death. Digital angiograms were then analysed by two independent, experienced interventional cardiologists, blinded to the data. All angiograms were assessed with respect to TIMI flow grade and TFC for the vessel in which the intervention was performed at baseline and after PCI.

\section{Determination of TIMI flow grade and TIMI frame count}

TIMI flow grades have been described previously as grade $0,1,2$, and 3 [10]. Coronary flow rates of all subjects were documented by TFC for each major coronary artery included in the study according to the method first described by Gibson et al. [9]. The left anterior descending coronary artery (LAD) is usually longer than the other major coronary arteries and the TFC for this vessel is often higher. To obtain a corrected TFC (CTFC) for the LAD, the TFC was divided by 1.7 . Intraobserver and interobserver variability for CTFC was $1.3 \pm 0.4$ and $2.1 \pm 0.6$ frames, respectively.

\section{Biochemical analyses}

Fasting blood samples were taken from all patients in the morning of the intervention day. Total cholesterol, triglyc- 
Table 1 Characteristics of the patients enrolled in the two groups

\begin{tabular}{|c|c|c|c|}
\hline & Statin group $(n=51)$ & Control group $(n=29)$ & $p$-value \\
\hline Age (years) & $58 \pm 4$ & $60 \pm 3$ & 0.3 \\
\hline Male gender, $n(\%)$ & $34(67)$ & $18(62)$ & 0.1 \\
\hline Left ventricular ejection fraction, $\%$ & $65 \pm 8$ & $65 \pm 5$ & 0.7 \\
\hline Current smokers, $n(\%)$ & $17(33)$ & $12(41)$ & 0.5 \\
\hline Systemic hypertension, $n(\%)$ & $26(50)$ & $16(55)$ & 0.1 \\
\hline Body mass index, $\mathrm{kg} / \mathrm{m}^{2}$ & $23 \pm 4$ & $25 \pm 4$ & 0.2 \\
\hline Family history of CAD & $24(47)$ & $14(48)$ & 0.4 \\
\hline Systolic BP, mm Hg & $120 \pm 5$ & $125 \pm 10$ & 0.1 \\
\hline $\begin{array}{l}\text { Diastolic BP, mm Hg } \\
\text { Heart rate, bpm }\end{array}$ & $\begin{array}{l}70 \pm 5 \\
76 \pm 6\end{array}$ & $\begin{array}{l}75 \pm 10 \\
78 \pm 8\end{array}$ & $\begin{array}{l}0.9 \\
02\end{array}$ \\
\hline $\begin{array}{l}\text { Number of diseased vessels, } n(\%) \\
1 \\
2 \\
3\end{array}$ & $\begin{array}{l}- \\
34(67) \\
12(23) \\
5(10)\end{array}$ & $\begin{array}{l}- \\
17(58) \\
9(31) \\
3(11)\end{array}$ & $\begin{array}{l}- \\
0.9 \\
0.1 \\
0.4\end{array}$ \\
\hline $\begin{array}{l}\text { Target vessel, } n(\%) \\
\text { LAD } \\
\text { RCA } \\
\text { LCX }\end{array}$ & $\begin{array}{l}- \\
25(49) \\
18(35) \\
8(16)\end{array}$ & $\begin{array}{l}- \\
14(48) \\
10(34) \\
5(18)\end{array}$ & $\begin{array}{l}- \\
0.2 \\
0.1 \\
0.8\end{array}$ \\
\hline Reference vessel diameter (mm) & $3.0 \pm 0.1$ & $3.2 \pm 0.2$ & 0.2 \\
\hline Target vessel stenosis, $\%$ & $80 \pm 10$ & $85 \pm 10$ & 0.09 \\
\hline Stent utilisation, $n(\%)$ & $51(100)$ & $29(100)$ & 0.4 \\
\hline Stent diameter $(\mathrm{mm})$ & $3.4 \pm 0.2$ & $3.5 \pm 0.3$ & 0.1 \\
\hline Stent length (mm) & $16 \pm 4$ & $18 \pm 5$ & 0.6 \\
\hline Dissection after PCI & $0(0)$ & $0(0)$ & 1.0 \\
\hline Residual stenosis, $\%$ & $15 \pm 5$ & $20 \pm 5$ & 0.3 \\
\hline Activated clotting time (s) & $280 \pm 10$ & $275 \pm 5$ & 0.5 \\
\hline Pre-PCI CTFC (frames/s) & $32 \pm 6$ & $35 \pm 5$ & 0.6 \\
\hline Post-PCI CTFC (frames/s) & $16 \pm 3$ & $22 \pm 5$ & 0.01 \\
\hline Post-PCI heart rate, bpm & $82 \pm 6$ & $80 \pm 8$ & 0.2 \\
\hline Post-PCI systolic BP, mm Hg & $125 \pm 10$ & $120 \pm 10$ & 0.1 \\
\hline Post-PCI diastolic BP, $\mathrm{mm} \mathrm{Hg}$ & $75 \pm 5$ & $80 \pm 5$ & 0.6 \\
\hline Total cholesterol (mmol/l) & $4.3 \pm 0.2$ & $4.1 \pm 0.3$ & 0.1 \\
\hline LDL cholesterol (mmol/l) & $2.4 \pm 0.6$ & $2.2 \pm 0.5$ & 0.1 \\
\hline HDL cholesterol (mmol/l) & $1.1 \pm 0.3$ & $1.0 \pm 0.2$ & 0.4 \\
\hline Triglycerides (mmol/l) & $1.5 \pm 0.2$ & $1.5 \pm 0.2$ & 0.6 \\
\hline hs-CRP (mg/l) & $2.1 \pm 0.7$ & $6.1 \pm 2$ & 0.01 \\
\hline Medical therapy, $n(\%)$ & - & - & - \\
\hline Nitrates & $51(100)$ & $29(100)$ & 1.0 \\
\hline ACE inhibitors & $14(27)$ & $8(28)$ & 0.7 \\
\hline Beta-blockers & $46(90)$ & $25(86)$ & 0.1 \\
\hline Calcium antagonists & $5(10)$ & $4(14)$ & 0.4 \\
\hline Aspirin & $51(100)$ & $29(100)$ & 1.0 \\
\hline $\begin{array}{l}\text { Glycoprotein IIb/IIIa receptor blocker administration } \\
\text { (tirofiban), } n(\%)\end{array}$ & $4(7)$ & $3(10)$ & 0.8 \\
\hline
\end{tabular}

$C A D$ coronary artery disease; $B P$ blood pressure; $L A D$ left anterior descending artery; $R C A$ right coronary artery; $L C X$ left circumflex artery; $P C I$ percutaneous coronary intervention; $C T F C$ corrected TIMI frame count; $L D L$ low-density lipoprotein; HDL high-density lipoprotein; $A C E$ angiotensin-converting enzyme; $h s$ - $C R P$ high-sensitivity $\mathrm{C}$-reactive protein

eride, high-density lipoprotein and low-density lipoprotein (LDL) levels were also measured by an auto analyser. Concentrations of high-sensitivity C-reactive protein (hs-CRP) were measured by latex-enhanced immunoturbidimetry.

\section{Statistical analysis}

Statistical analysis was performed using the SPSS statistical package (SPSS, Inc., Chicago, Illinois). Continuous variables were expressed as mean \pm standard deviation and categorical variables as $\%$. While the chi-square 


\section{CTFC}

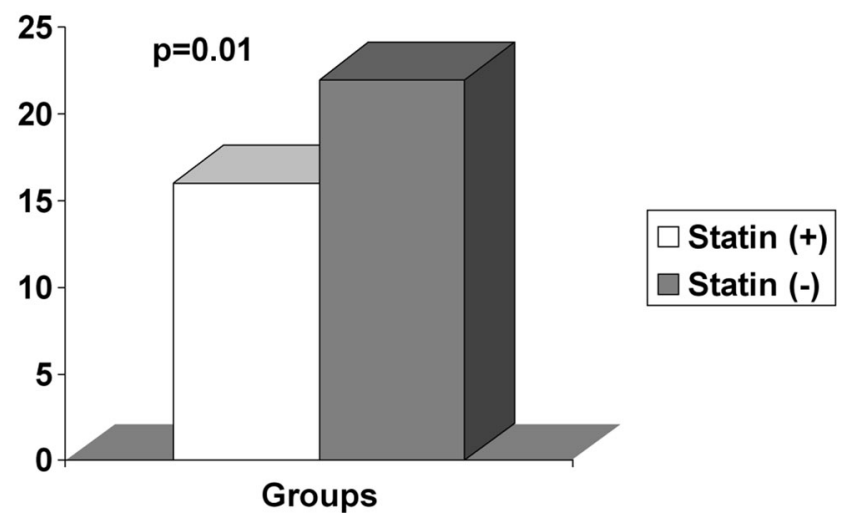

Fig. 1 The comparison of corrected TIMI frame count (CTFC) between two groups

test or Fisher's exact test was used for categorical values between the two groups, differences between groups in normally and non-normally distributed variables were assessed using the unpaired Student's t test and the Mann-Whitney $\mathrm{U}$ test, respectively. Analysis of covariance (ANCOVA) was used to analyse the confounding effects of variables on the comparisons of the groups according to statin use before PCI. The multivariate analysis was used to define independent factors influencing CTFC after PCI. The following selected variables were inserted into the multivariate analysis: statin pre-treatment, age, body mass index, family history of CAD, systolic and diastolic blood pressure, hsCRP level, hypertension, concomitant medications, smoking, serum total cholesterol, LDL-cholesterol levels, lesion length, residual percent stenosis, vessel diameter, and vessel type. A $p$ value of $<0.05$ was considered statistically significant.

\section{Results}

The characteristics of the study population are listed in Table 1 . There was no statistically significant difference in the baseline characteristics analysed, which included patient age, sex, body mass index, left ventricular ejection fraction, systolic and diastolic blood pressure, hypertension, smoking, family history of CAD and various medication used. Lipid parameters were also comparable between the two groups (Table 1).

All the angiographic and procedural characteristics were similar in the two groups (Table 1). A final TIMI 3 flow was achieved in all patients. Angiographic complications during the procedure did not occur. It reached the target ACT levels during PCI in all patients. Glycoprotein $\mathrm{IIb} /$ IIIa inhibitors were administered in 4 of 51 patients $(7.0 \%)$ in the statin group and in 3 of 29 patients (10\%) in the control group $(p=0.8)$. Intracoronary vasodilator agents were not used.

Although pre-PCI CTFC values were similar between the two groups ( $32 \pm 6$ vs. $35 \pm 5, p=0.6$ ), post-PCI CTFC in patients treated with statin before PCI was significantly lower than the control group ( $16 \pm 3$ vs. $22 \pm 5, p=0.01$, Fig. 1). The hs-CRP level was significantly lower in the patients taking statin in compared with the control group $(2.1 \pm 0.7 \mathrm{mg} / \mathrm{l}$ vs. $6.1 \pm 2 \mathrm{mg} / \mathrm{l}, p=0.01)$ (Table 1$)$. No significant differences were found between the two groups for any of the other analysed variables. Multiple logistic regression analysis showed that only statin pre-treatment (OR 2.5, $95 \%$ CI 1.2 to $3.8, p<0.001$ ) and hs-CRP level (OR $1.8,95 \%$ CI 1.2 to $2.4, p=0.001$ ) were independent predictors of post-PCI CTFC.

\section{Discussion}

This study showed that receipt of chronic statin therapy before PCI in patients with stable CAD is associated with decreased CTFC of the target vessel, suggesting the improvement of microvascular function.

Conventional TIMI flow grading is a predictor of cardiac outcome after acute myocardial infarction and PCI, but it has several limitations [11]. The CTFC, another approach to grade flow impairment, is an objective, quantitative, reproducible, and sensitive index for coronary blood flow [9]. As indicated in our study, TIMI flow may appear normal visually, but may correlate to abnormal CTFC. The CTFC has been proposed to have incremental prognostic accuracy in predicting survival outcome with reperfusion therapy [12]. This measurement was significantly correlated with flow velocity measured with FloWire by several investigators during baseline conditions or hyperaemia [13]. So the CTFC may be an index of microvascular behaviour, which reflects coronary vascular resistance [14]. Higher CTFC values after PCI have also been found to be associated with poor clinical outcomes [15]. In addition to these studies using thrombolysis or balloon angioplasty, in which higher values of CTFC were associated with adverse clinical outcome, there are several studies regarding CTFC's relevance in the current stent era [16-19]. In this study, the post-PCI CTFC in patients receiving statin before PCI was significantly lower than those of the patients not receiving statins. This finding may be attributed to the potential beneficial effects of statins on coronary microcirculation.

Lipid profiles were similar between the two groups in our study; therefore, the beneficial effects observed may be considered independent of cholesterol lowering. Although the present study was not designed to elucidate the potential mechanisms of the cardioprotective effect of the statins, some possible mechanisms can be hypothesised. It is sug- 
gested that improvement of myocardial vascular function by statins is not only a result of reduction in plasma lipids but is also caused by some action on blood vessels other than a lipid-lowering effect (so-called pleiotropic effects) [5]. The pleiotropic effects encompass non-lipid mechanisms that modify endothelial function, inflammation responses, plaque stability, and thrombus formation [20, 21]. Among those effects, improvement in endothelial function, anti-thrombotic and anti-inflammatory actions may be crucial for restoration of coronary blood flow after PCI, because those effects are consistent with a possible underlying mechanism of slow coronary flow [22]. As in coronary instability, vascular injury during PCI is associated with a systemically measurable inflammatory response and the degree of inflammation has been shown to be correlated with cardiovascular risks [23, 24]. The improvement of coronary blood flow associated with statin therapy might be explained based on its anti-inflammatory effects, but with the present evidence, this remains speculative.

Egashira et al. [25] demonstrated the improvement of endothelium-dependent coronary vasomotion using acetylcholine-stressed quantitative coronary arteriography and coronary flow velocity reserve using an intracoronary Doppler catheter after 2 and 6 months of pravastatin treatment in patients with dyslipidaemia. They speculated that impaired endothelium-dependent vasomotion may play a role in modulating myocardial perfusion in patients with hypercholesterolaemia, typical-effort angina pectoris, and critical stenosis of the epicardial coronary artery. It was also found that pravastatin increased microvascular perfusion in normocholesterolaemic patients with single-vessel disease after successful PCI [26]. Briguori et al. [27] showed that atorvastatin $80 \mathrm{mg}$ loaded 24 hours prior to PCI in statin-naive patients significantly reduced the risk of periprocedural myocardial infarction as defined by elevations of creatine-kinase myocardial enzyme and cardiac troponin I. At 30 days, high-dose statin therapy reduced the risk of periprocedural myocardial infarction by $44 \%$, defined by both markers. The findings from NAPLES II support the need to postpone the intervention in statinnaive patients undergoing an elective procedure. These findings may be attributed to the potential beneficial effects of statins on $\mathrm{CBF}$ after PCI.

\section{Limitations}

There are several limitations of this study. First, it was a retrospective study with a small number of patients. Larger multicentre studies are needed to corroborate our findings. Secondly, we could not estimate differences in efficacy among the various statins because of the relatively small numbers of patients in the study. There were substantial differences in CTFC pre- and post-PCI in this study. This would indicate that resting flow was impaired prior to PCI. Resting flow usually remains constant up to subtotal lesions. A proximal stenosis must generally exceed $75 \%$ diameter narrowing before its resistance approximates that of the resting coronary vascular bed resistance and begins to reduce resting flow. In our study population, mean percent diameter stenosis was 80 and $85 \%$ respectively. This might indicate that resting flow was impaired prior to PCI. The final limitation was the impossibility of follow-up, which could have informed us concerning the long-term clinical outcomes.

\section{Conclusions}

In conclusion, the present study showed that statin therapy before PCI in patients with stable CAD may have some beneficial effects on coronary blood flow demonstrated by CTFC. Further large-scale prospective randomised clinical trials are needed to elucidate the underlying mechanisms and clinical importance of these findings.

\section{Compliance with ethical guidelines}

Conflict of interest L. Cerit, H. Duygu, K. Gulsen and A. Gunsel state that he/she/they have/has no competing interest.

This article does not contain any studies with human participants or animals performed by any of the authors.

Open Access This article is distributed under the terms of the Creative Commons Attribution 4.0 International License (http:// creativecommons.org/licenses/by/4.0/), which permits unrestricted use, distribution, and reproduction in any medium, provided you give appropriate credit to the original author(s) and the source, provide a link to the Creative Commons license, and indicate if changes were made.

\section{References}

1. Scandinavian Simvastatin Survival Study Group. Randomized trial of cholesterol lowering in 4444 patients with coronary heart disease: the Scandinavian Simvastatin Survival Study (4S). Lancet. 1994;344:1383-9.

2. Long-term Intervention with Pravastatin in Ischemic Disease (LIPID) study group. Prevention of cardiovascular events and death with pravastatin in patients with coronary heart disease and a broad range of initial cholesterol levels. N Engl J Med. 1998;339:1349-57.

3. Schwartz GG, Olsson AG, Ezekowitz MD, et al. Myocardial Ischemia Reduction with Aggressive Cholesterol Lowering (MIRACL) Study Investigators. Effects of atorvastatin on early recurrent ischemic events in acute coronary syndromes: the MIRACL study. A randomized controlled trial. JAMA. 2001;285:1711-8.

4. Stenestrand U, Wallentin L. Early statin treatment following acute myocardial infarction and 1-year survival. JAMA. 2001;285:430-6. 
5. Takemoto M, Liao JK. Pleiotropic effects of 3-hydroxy-3-methylglutaryl coenzyme A reductase inhibitors. Arterioscler Thromb Vasc Biol. 2001;21:1712-9.

6. Baller D, Notohamiprodjo G, Gleichmann U, Holzinger J, Weise R, Lehmann J. Improvement in coronary flow reserve determined by positron emission tomography after 6 months of cholesterol-lowering therapy in patients with early stages of coronary atherosclerosis. Circulation. 1999;99:2871-5.

7. Kaesemeyer WH, Caldwell RB, Huang J, Caldwell RW. Pravastatin sodium activates endothelial nitric oxide synthase independent of its cholesterol-lowering actions. J Am Coll Cardiol. 1999;33:234-41.

8. Sposito AC, Chapman J. Statin therapy in acute coronary syndromes. Mechanisms insight into clinical benefit. Arterioscler Thromb Vasc Biol. 2002;22:1524-34.

9. Gibson CM, Cannon CP, Daley WL, et al. TIMI frame count: a quantitative method of assessing coronary artery flow. Circulation. 1996;93:879-88.

10. The TIMI Study Group. Thrombolysis in myocardial infarction (TIMI) trial. N Engl J Med. 1985;312:932-6.

11. Simes RJ, Topol EJ, Holmes DR Jr, et al. Link between the angiographic substudy and mortality outcomes in a large randomized trial of myocardial reperfusion. Importance of early and complete infarct artery reperfusion. Circulation. 1995;91:1923-8.

12. Gibson CM, Goel M, Cohen DJ, et al. Six-month angiographic and clinical follow-up of patients prospectively randomized to receive either tirofiban or placebo during angioplasty in the RESTORE trial: Randomized Efficacy Study of Tirofiban for Outcomes and Restenosis. J Am Coll Cardiol. 1998;32:28-34.

13. Kern MJ, Moore JA, Aguirre FV, et al. Determination of angiographic (TIMI grade) blood flow by intracoronary Doppler flow velocity during acute myocardial infarction. Circulation. 1996;94:1545-52.

14. Manginas A, Gatzov P, Chasikidis C, Voudris V, Pavlides G, Cokkinos DV. Estimation of coronary flow reserve using the thrombolysis in myocardial infarction (TIMI) frame count method. Am J Cardiol. 1999;83:1562-5.

15. Gibson CM, Murphy SA, Rizzo MJ, et al. Relationship between TIMI frame count and clinical outcomes after thrombolytic administration. Circulation. 1999;99:1945-50.

16. Jespersen L, Abildstrøm SZ, Peña A, et al. Predictive value of the corrected TIMI frame count in patients with suspected angina pec- toris but no obstructive coronary artery disease at angiography. Clin Res Cardiol. 2014;103:381-7.

17. Bencze J, Kiss RG, Toth-Zsamboki E, et al. Inverse correlation between coronary blood flow velocity and sICAM-1 level observed in ischemic heart disease patients. Atherosclerosis. 2006;188:142-9.

18. Lyu T, Zhao Y, Zhang T, et al. Effect of statin pretreatment on myocardial perfusion in patients undergoing primary percutaneous coronary intervention: a systematic review and meta-analysis. Clin Cardiol. 2013;36:E17-24.

19. Jia XW, Fu XH, Zhang J, et al. Intensive cholesterol lowering with statin improves the outcomes of percutaneous coronary intervention in patients with acute coronary syndrome. Chin Med J. 2009;122:659-64.

20. Swenne CA. Beyond lipid lowering: pleiotropic effects of statins in heart failure. Neth Heart J. 2013;21:406-7.

21. On behalf of the CCR Study Investigators, Yetgin T, van der Linden MM, de Vries AG, et al. Current discharge management of acute coronary syndromes: data from the Rijnmond Collective Cardiology Research (CCR) study. Neth Heart J. 2014;22:20-7.

22. Li JJ, Zheng X, Li J. Statins may be beneficial for patients with slow coronary flow syndrome due to its anti-inflammatory property. Med Hypotheses. 2007;69:333-7.

23. Gaspardone A, Crea F, Versaci F, et al. Predictive value of C-reactive protein after successful coronary-artery stenting in patients with stable angina. Am J Cardiol. 1998;82:515-8.

24. Chew DP, Bhatt DL, Robbins MA, et al. Incremental prognostic value of elevated baseline $\mathrm{C}$-reactive protein among established markers of risk in percutaneous coronary intervention. Circulation. 2001;104:992-7.

25. Egashira K, Hirooka Y, Kai H, et al. Reduction in serum cholesterol with pravastatin improves endothelium-dependent coronary vasomotion in patients with hypercholesterolemia. Circulation. 1994;89:2519-24.

26. Manfrini O, Pizzi C, Morgagni G, Fontana F, Bugiardini R. Effect of pravastatin on myocardial perfusion after percutaneous transluminal coronary angioplasty. Am J Cardiol. 2004;93:1391-3.

27. Briguori C, Visconti G, Focaccio A, et al. Novel approaches for preventing or limiting events (naples) II trial impact of a single high loading dose of atorvastatin on periprocedural myocardial infarction. J Am Coll Cardiol. 2009;54:2157-63. 